\title{
Peel and peel again
}

\author{
S K Gibran, ${ }^{1}$ B Flemming, ${ }^{1}$ T Stappler, ${ }^{1}$ I Pearce, ${ }^{1}$ C Groenewald, ${ }^{1}$ H Heimann, ${ }^{1}$ \\ P Hiscott, ${ }^{1}$ D Wong ${ }^{1,2}$
}

${ }^{1}$ St Paul's Eye Unit, Royal Liverpool University Hospital and Unit of Ophthalmology, University of Liverpool, UK; ${ }^{2}$ Li Ka Shing Faculty of Medicine, University of Hong Kong, Hong Kong SAR,

China

Correspondence to:

S K Gibran, St Paul's Eye Unit, Royal Liverpool University

Hospital, Prescot Street,

Liverpool L7 8XP, UK;

syedgibran@yahoo.com

Accepted 31 October 2007

Published Online First

30 November 2007

\section{ABSTRACT}

Aim: To determine if the internal limiting membrane (ILM) was present in the epiretinal membrane (ERM) when we deliberately tried to perform a "double peel" for macular pucker.

Methods: Pars-plana vitrectomy and a "double peel" were carried out. The ERM and ILM were stained with Trypan Blue and peeled separately over the same area. The amount of ERM present in ILM specimens and the amount of ILM present in ERM specimens were evaluated by histological examination.

Results: Seventeen eyes in 17 patients were included. It was possible to double peel in all cases. Five of 17 ERM specimens $(29 \%)$ contained ILM fragments. When ILM was present on the ERM, it represented less than $50 \%$ of the sample. One ILM specimen was lost as result of an administrative error; of the remaining 16 specimens, residual ERM was found in six, and cellular remnants were observed on the vitreous surface in a further six of the ILMs. Clinically, no recurrence of ERM was found.

Conclusion: ILM was present in some ERM specimens seemingly over the same area that an intact ILM was subsequently peel. We speculate that the ILM in the ERM represent a secondary basement membrane and that the surgical plane of dissection for most ERM peel is between the ERM and the native ILM, making it feasible to double peel routinely.

Peeling of the internal limiting membrane (ILM) for treatment of macular hole is established, ${ }^{12}$ whereas peeling of ILM in macular pucker surgery remains more controversial. ${ }^{3}$ Theoretically, the advantages for ILM peel for macular pucker would include a more complete removal of the epiretinal membrane (ERM). There is evidence that denuding the retina of ILM over a large area might also inhibit reproliferation. ${ }^{5}$ Park et $a l,{ }^{6}$ in a randomised study, showed that peeling of ILM during macular pucker surgery might not have a deleterious effect. On the other hand, there is no evidence from randomised trials to show that peeling of ILM leads to a better anatomical and functional result or reduces the rate of recurrence of ERM. Despite this lack of evidence, double peeling seems to be becoming more popular. Does this practice have a sound histological basis? We are intrigued by the fact that prior to double peeling being performed routinely, there have been many published reports on the histology of the ERM. These showed that a good proportion of the excised ERM specimens contain ILM ${ }^{5}$ and. . If the ERM contains ILM, how is it possible to peel again and remove another ILM? Are there truly two ILM in some cases? If so, how does it come about? Is there any good histological evidence to suggest that removal of the ILM gives rise to a more complete removal of the ILM?
This study sets out to perform a clinicopathological correlate to determine whether the histology confirms that ILM can be present in both ERM and ILM specimens when they are harvested from the same area.

\section{PATIENTS AND METHODS}

This study was a prospective, consecutive interventional case series. Patients scheduled for primary idiopathic and secondary ERM surgery in St Paul's Eye Unit, Royal Liverpool University Hospital, UK were included. Individuals over 16 years of age requiring vitrectomy for ERM removal were recruited prospectively. Only first-time vitreous surgery was included. Preoperative data including age, sex, logMar BCVA and co-morbidity were collected. Postoperative logMar BCVA, anatomical outcome, and histopathological findings were recorded. The aetiology of the macular pucker was previous retinal detachment, ${ }^{3}$ proliferative diabetic retinopathy ${ }^{4}$ and idiopathic. ${ }^{10}$

\section{Intervention}

A three-port pars-plana vitrectomy was performed in all patients. Posterior vitreous detachment was induced where necessary. A volume of $0.2-0.3 \mathrm{ml}$ of Trypan Blue (Membrane blue ${ }^{\mathrm{TM}}$, DORC International bv, Zuidland, The Netherlands) was used. The solution was injected into the air-filled vitreous cavity over the posterior pole. After $2 \mathrm{~min}$, still under air, the dye was removed using a back flush needle. An air/fluid exchange was then carried out. The ERM was stained a faint blue colour and was clearly visible under standard illumination. The blue stained tissue was directly engaged with the intraocular micro-forceps (Eckardt End Gripping Forceps, DORC International bv, Zuidland, The Netherlands) to create a flap and then peeled from the retinal surface. After ERM removal, TB solution was injected once again under air for another $2 \mathrm{~min}$ to stain the ILM. The ILM was peeled in a capsulorrhexis fashion and removed as a sheet. No tamponade agents were used. The specimens were retrieved according to the technique described by the authors, ${ }^{8}$ which enabled the small excised ERM and ILM specimens to be individually monitored throughout the histological processing procedure. The method ensures a low rate of specimen loss.

\section{Light microscopy}

Specimens were fixed in 10\% neutral buffered formalin, dehydrated in graded concentrations of ethanol and embedded in paraffin wax. Sections of wax embedded tissue were cut, dewaxed and 
stained with haematoxylin and eosin. Further sections were stained with the periodic acid Schiff (PAS) method. A record was made of the morphological features of the excised tissue, the presence or absence of ILM and of cells or cell remnants on the ILM. An estimate of the proportion of ERM and ILM in the tissue was made as previously described. ${ }^{9}$

The vitreal surface of the ILM is smooth, and the retinal surface is irregular (undulating). ${ }^{10}$ Thus, it was possible to determine whether cells or cell remnants were attached to vitreous or retinal surfaces of the ILM.

\section{RESULTS}

We report the results of 17 eyes of 17 patients. Eleven patients were male, and six were female. The mean age was 57.9 years (range 32-74). Preoperative logMar BCVA ranged from 0.5 to 1.0 (mean 0.8). Postoperative logMar BCVA ranged from 0 to 1.0 (mean 0.4) $(p<0.001)$. Vision was improved or was maintained in all $17(100 \%)$ patients. The mean follow-up period was 9.4 months (range 6-12 months). At the last followup, on biomicroscopy, none of the 17 cases showed obvious signs of residual membranes or recurrence of pucker. In all 17 eyes, the ERM and ILM were adequately stained and visualised during surgery. No intraoperative or postoperative complications relating to the use of the dye or to the double peeling were observed. Surgically, it was possible to double peel all cases. This applies to the early diaphanous ERM as well as the thicker late ERM. The surgically excised tissues from the first peel were labelled specimen-A and from the second peel specimen-B for histological study.

First peel specimens were retrieved from 17 of the 17 eyes. Second peel specimens were retrieved from 17 of 17 eyes, but one specimen was lost as a result of administrative error, such that only 16 were available for histological analysis. Detailed histological and clinical findings have been elaborated in table 1. First peel specimen consisted principally of ERM accounting for nearly $100 \%$ of the tissue in most cases. Likewise, second peel specimens were made up ILM that represented nearly $100 \%$ of the tissue in many cases. About a third of first peel specimen (five of the 17) contained ILM (figs 1 and 2). When ILM was present, it usually occupied only a small fraction of the specimen (10-30\%); in one first peel ERM specimen, however, ILM represented $50 \%$ of the excised tissue (table 1). ERM was found in conjunction with ILM in just over a third of second peel specimens (six of the 16). The ERM in second peel specimens generally consisted of incomplete layers of cells, but in one case a fragment of fibrocellular ERM represented approximately $30 \%$ of the tissue (table 1 , fig 1 ). In addition, isolated cells or small cell foci were found on the vitreous (smooth) surface of the ILM in a further six ILM specimens. All ILM specimens had cell remnants adherent to the retinal (undulating) surface of the ILM.

\section{DISCUSSION}

At the time of surgery, ERM can have varied appearances, ranging from being translucent, thin, pliable and diaphanous to being white, thick, stiff and fibrous depending on the nature and the duration of the underlying eye condition. ILM on the other hand, are usually transparent and uniform in thickness, and the membrane has a tendency to scroll. Whilst it is possible to distinguish between ERM and ILM, it is clinically difficult to tell just by the appearance at surgery whether a membrane is an ERM or an ERM with an ILM. After removal of the ERM, there is no easy way of telling whether it is safe to perform a second peel of ILM. In other words, without histological confirmation, surgeons cannot be confident of the surgical dissection plane of the ERM peel. Their uncertainty is added to by the literature, which showed that the histology of ERM often contains ILM.

Kwok used ICG for double peeling. ${ }^{5}$ ICG has an affinity for the ILM and may be more specific than other dyes in staining the ILM. In situations where the surgeons are uncertain if the ILM was removed in part or completely, it made sense to use ICG. There are, however, serious concerns about the possible toxic effect of ICG. ${ }^{12}{ }^{13} \mathrm{~TB}$ is not specific in that it stains both ERM and ILM. In the past, as in this study, we have found it to be helpful in peeling the ILM. ${ }^{14}$

Table 1 Patients' demographics, clinical features and histological findings

\begin{tabular}{|c|c|c|c|c|c|c|c|c|c|c|c|}
\hline \multirow[b]{2}{*}{$\mathbf{P t}$} & \multirow[b]{2}{*}{$\begin{array}{l}\text { Age/ } \\
\text { sex }\end{array}$} & \multirow[b]{2}{*}{$\begin{array}{l}\text { Preop } \\
\text { VA }\end{array}$} & \multirow[b]{2}{*}{$\begin{array}{l}\text { Postop } \\
\text { VA }\end{array}$} & \multirow[b]{2}{*}{ Aetiology } & \multicolumn{3}{|c|}{ ERM specimen (A) } & \multicolumn{4}{|c|}{ ILM specimen (B) } \\
\hline & & & & & $\begin{array}{l}\text { ERM (\% } \\
\text { tissue) }\end{array}$ & (Type) & $\begin{array}{l}\text { ILM* } \\
\text { (\% tissue) }\end{array}$ & $\begin{array}{l}\text { ERM } \\
\text { (\% tissue) }\end{array}$ & (Type) & $\begin{array}{l}\text { ILM* } \\
\text { (\% tissue) }\end{array}$ & (Vitreous surface) \\
\hline 1 & $64 / \mathrm{M}$ & 0.8 & 0.0 & Idiopathic & $+(100)$ & $(\mathrm{F} / \mathrm{C})$ & - & - & & $+(100)$ & \\
\hline 2 & $74 / \mathrm{M}$ & 1.0 & 0.3 & Idiopathic & $+(50)$ & $(\mathrm{F} / \mathrm{C})$ & $+(50)$ & - & & $+(100)$ & (Cell remnants) \\
\hline 3 & $58 / \mathrm{M}$ & 0.6 & 0.0 & Idiopathic & $+(70)$ & $(\mathrm{F} / \mathrm{C})$ & $+(30)$ & - & & $+(100)$ & (Cell remnants) \\
\hline 4 & $54 / \mathrm{M}$ & 0.5 & 0.3 & Idiopathic & $+(100)$ & $(F / C, V)$ & - & $+(10)$ & (Incomplete simple $\dagger$ ) & $+(90)$ & \\
\hline 5 & $64 / \mathrm{M}$ & 0.6 & 0.5 & Idiopathic & $+(100)$ & $(F / C)$ & - & - & & $+(100)$ & \\
\hline 6 & $70 / F$ & 0.8 & 0.0 & Idiopathic & $+(90)$ & $(\mathrm{F} / \mathrm{C})$ & $+(10)$ & - & & $+(100)$ & (Cell remnants) \\
\hline 7 & $66 / \mathrm{M}$ & 0.6 & 0.0 & Idiopathic & $+(50)$ & $(\mathrm{F} / \mathrm{C})$ & $+(30)$ & $+(10)$ & (Incomplete simple $\dagger$ ) & $+(90)$ & \\
\hline 8 & $58 / F$ & 0.6 & 0.2 & Idiopathic & $+(90)$ & $(\mathrm{F} / \mathrm{V})$ & $+(10)$ & No tissue & & & \\
\hline 9 & $62 / \mathrm{M}$ & 0.8 & 0.3 & Idiopathic & $+(100)$ & $(F / C ; V)$ & - & $+(30)$ & $(F / C ; V)$ & $+(70)$ & \\
\hline 10 & $56 / F$ & 0.5 & 0.5 & Idiopathic & $+(100)$ & $(F / C)$ & - & - & & $+(100)$ & \\
\hline 11 & $62 / F$ & 1.0 & 0.8 & PVR & $+(100)$ & $(F / C)$ & - & $+(10)$ & (Incomplete simple $\dagger$ ) & $+(90)$ & \\
\hline 12 & $64 / F$ & 1.0 & 0.8 & PVR & $+(100)$ & $(F / C)$ & - & - & & $+(100)$ & (Cell remnants) \\
\hline 13 & $67 / F$ & 1.0 & 0.8 & PVR & $+(100)$ & $(\mathrm{F} / \mathrm{C})$ & - & - & & $+(100)$ & (Isolated cells) \\
\hline 14 & $32 / \mathrm{M}$ & 1.0 & 1.0 & PDR & $+(100)$ & $(F / V)$ & - & - & & $+(100)$ & (Cell foci) \\
\hline 15 & $38 / \mathrm{M}$ & 1.0 & 1.0 & PDR & $+(100)$ & $(F / N)$ & - & $+(10)$ & (Incomplete simple $\dagger$ ) & $+(90)$ & \\
\hline 16 & $48 / \mathrm{M}$ & 1.0 & 0.8 & PDR & $+(100)$ & $(F / N)$ & - & - & & $+(100)$ & \\
\hline 17 & $48 / \mathrm{M}$ & 1.0 & 0.8 & PDR & $+(100)$ & $(F / V)$ & - & $+(10)$ & $(F / C)$ & $+(90)$ & \\
\hline
\end{tabular}

${ }^{*}$ All ILM had cell remnants on the retinal surface.

†Simple ERM: cell monolayer. ${ }^{11}$

ERM, epiretinal membrane; F/C, fibrocellular; F/N, fibrovascular; ILM, internal limiting membrane; PDR, proliferative diabetic retinopathy; Pt, patient; PVR, proliferative vitreoretinopathy; V, vacuolated cells; VA, logMar VA. 
Figure 1 Sections through ERM ( $A$ and $\mathrm{B})$ and corresponding ILM (C-F), and an ILM from a different patient $(G, H)$, stained with haematoxylin and eosin $(A$, $C, E, G$ ) or periodic acid-Schiff reagent with haematoxylin counterstain (B, D, F, $\mathrm{H})$. In the specimens from the first patient, no ILM is seen within the fibrocellular ERM specimen (A, B). The corresponding ILM specimen (C-F) does not contain fibrocellular ERM. However, isolated cells or small cell foci are seen on the vitreous (smooth) surface of the ILM (circles in $C$ and $D$, arrows in $E$ and $F$ : the areas within the circles in $C$ and $D$ can be seen at a higher magnification in $\mathrm{E}$ and $\mathrm{F}$ ). The ILM specimen also shows cell remnants adherent to the retinal (undulating) surface of the ILM (arrowheads, E and F). G and $\mathrm{H}$ demonstrate that the ILM from another patient has a fragment of fibrocellular epiretinal membrane (Ep) within the specimen. Original magnifications: A-D and $G, H: \times 100 ; E, F: \times 400$.
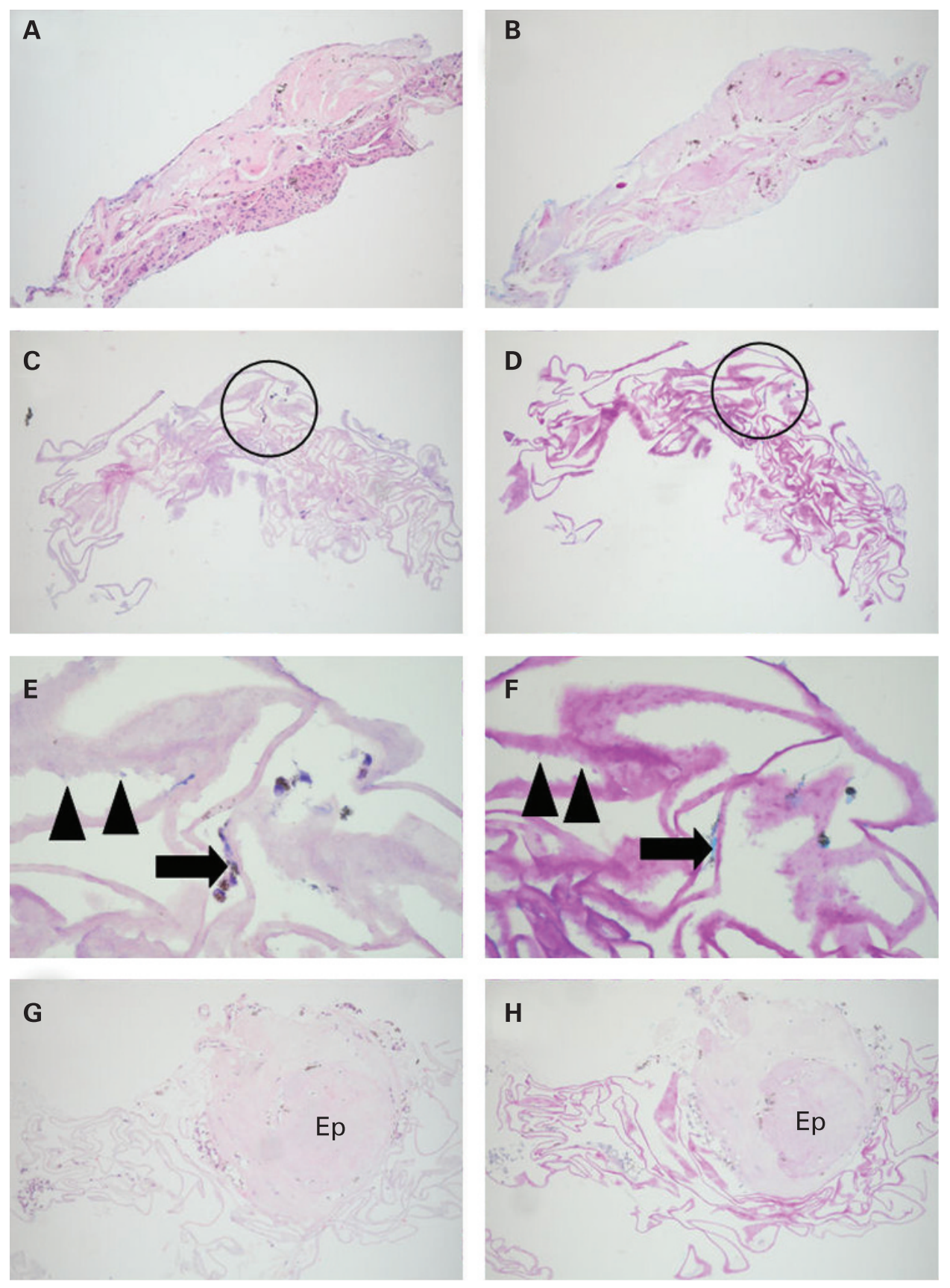

The method of peeling ERM in this study was standard using end-gripping forceps to engage the membrane. We had not modified our technique because we intended to perform a double peel. We were impressed that in every case, it was possible to peel the ILM as a separate layer. We were further struck by the fact that in every case, the ILM was an intact sheet, with no defects or focal damage as a result of the ERM peel. Once an edge was identified, the ILM was peeled in much the same way as one would in macular hole surgery that is as an intact sheet. There were no indications that part of the lamina has been removed with the ERM. This was not only our experience but that of others. ${ }^{5}{ }^{6}$

Histologically, we found that ILM was present in some specimens of ERM. Our results showed that only five of 17 ERMs had elements or fragments of ILM, and the amount varied from 10 to a maximum of $50 \%$. Yet surgically, it was possible to remove the ILM as a separate and complete layer in each of the five cases. How is it possible for there to be ILM on both specimens?

A possible though unlikely explanation might be that the specimens were from different parts of the retina. The surgery was often recorded on video, and on reviewing the surgery we were absolutely convinced that we peeled the ILM over the same area. Another possible explanation is that there is schisis of the ILM such that we have a complete lamina of ILM left on the retina after ERM peel. This would mean that the surgical plane is inconsistent. Because there are often fragments of ILM, at these areas or points the surgical plane must have been within the ILM itself. However, another observation that we made was that the ILM fragments attached to ERM histologically have an irregular surface (fig 1). If there had been schisis within the ILM, one might have expected a schitic ILM to be 
Figure 2 Sections through ERM (A and $B)$ and ILM (C and D) removed during the same surgical procedure. A small fragment of ILM can be seen with the ERM specimen (arrows), whereas the ILM is devoid of epiretinal tissue. ( $A$ and C; haematoxylin and eosin stain: B and D periodic acid-Schiff reagent with haematoxylin counterstain.) Original magnifications: $\mathrm{A}-\mathrm{D}: \times 150$.
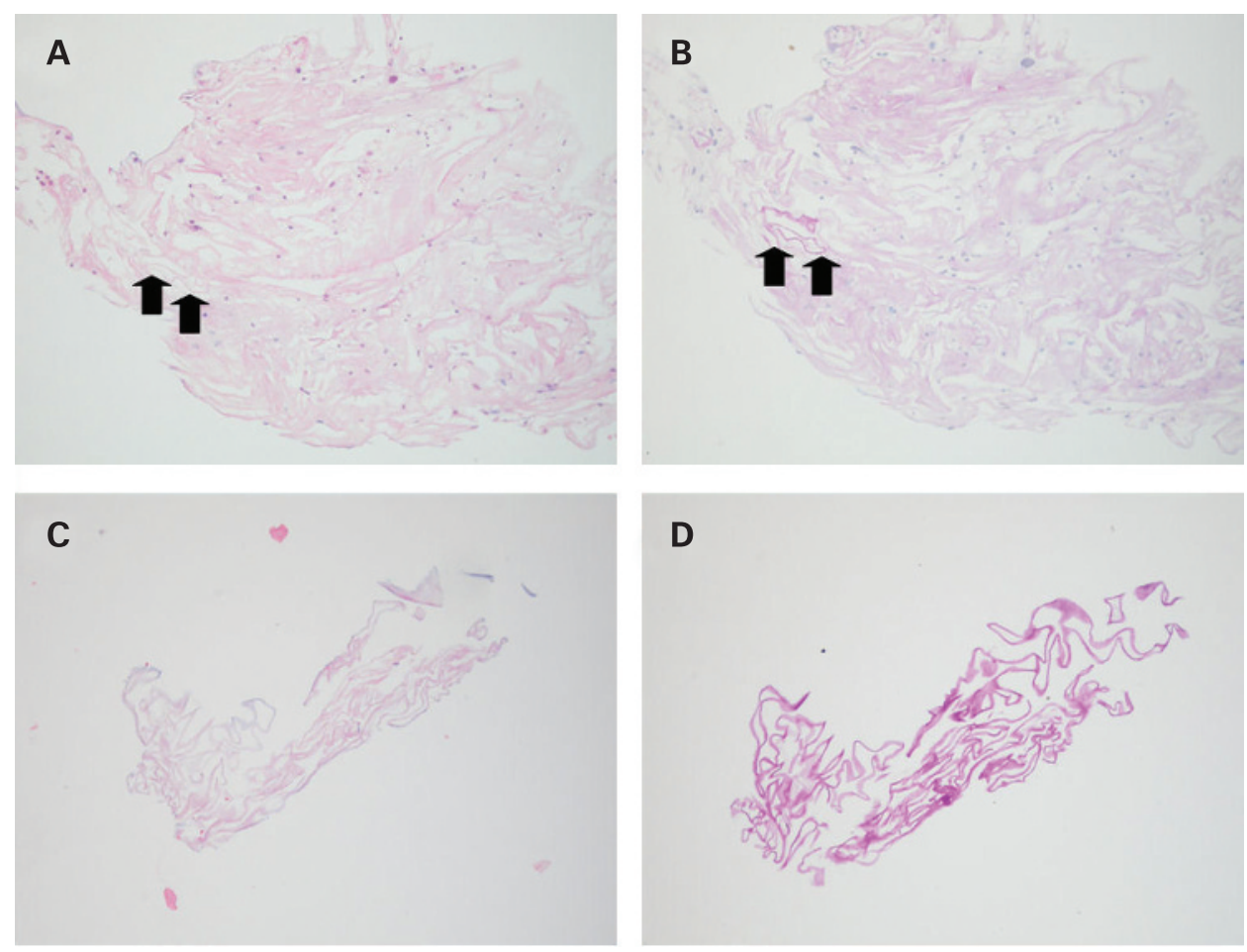

smooth on each surface (as in fig 3A). Partial separation of the ERM from the retina is not unusual and is often detected on preoperative optical coherence tomography (OCT). Wilkins et $a l^{15}$ examined ERM in 186 eyes of 160 patients with OCT and found that the ERM in 49 eyes had clearly separated from the retina. We envisage that when an ERM separates from the retinal surface a cleft is formed in one of two ways. Either a fragment of ILM detaches with the ERM (as in fig 3B), in which case there would be holes or defects in the native ILM. It could be that the footplates of Muller cells subsequently laid down new basement membrane to reform ILM and fill in these holes or defects (as in fig 3C). There is, however, little evidence in the literature for repair or reformation of ILM after removal. For example, no one has ever reported the peeling of ILM twice and
Figure 3 (A) Diagrammatic representation of ILM schisis. (B) Focal spontaneous separation of ERM with avulsion of ILM fragment underneath. (C) Subsequent reproliferation leads to secondary ILM on the surface of retina. (D) Focal spontaneous separation of ERM with avulsion of ILM fragment underneath. (E) Subsequent reproliferation leads to secondary ILM-like structure on the retinal side of ILM.

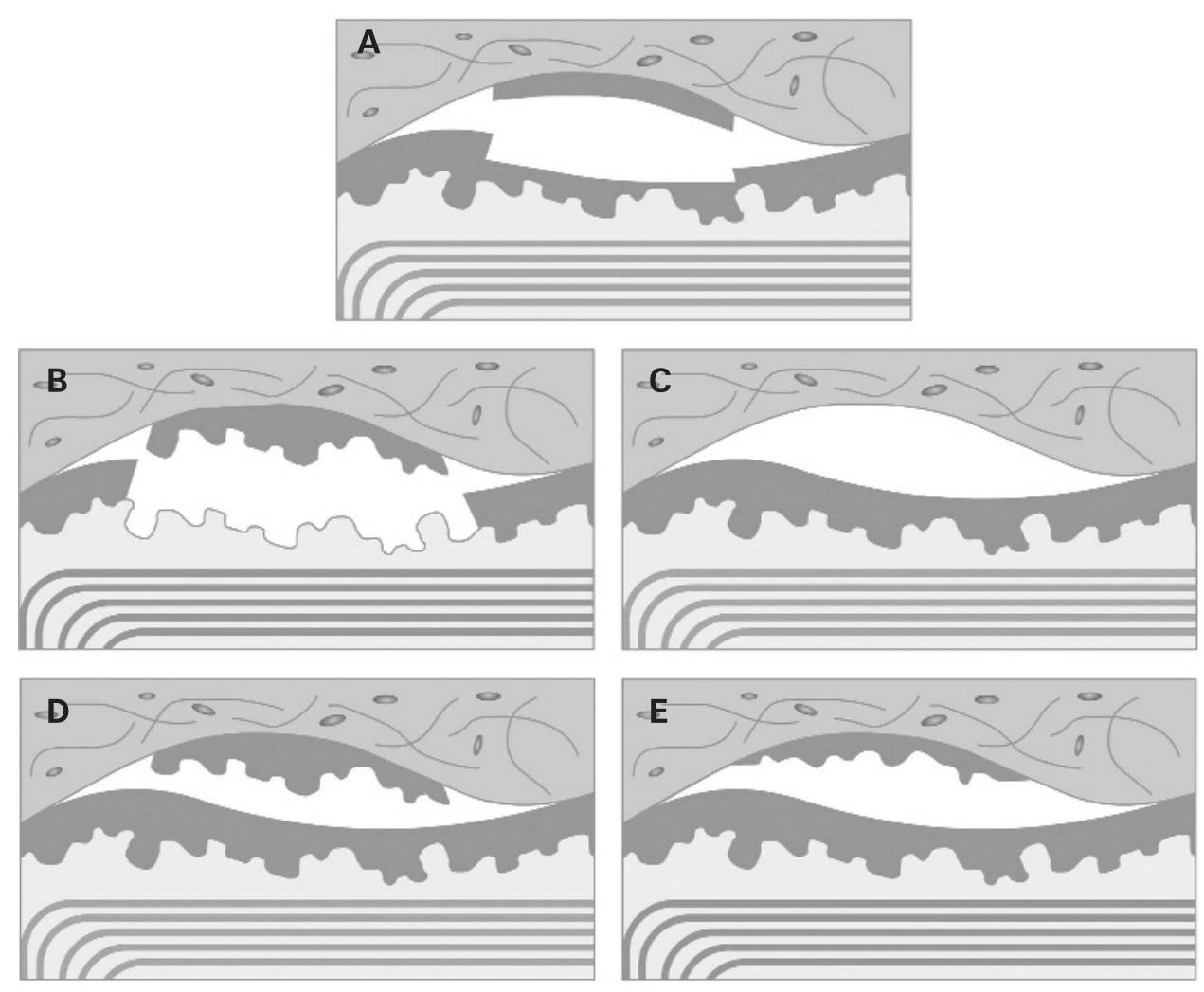


over the same area even with ICG staining during repeat vitrectomy for failed macular hole surgery. The alternative is that the ERM detaches leaving the native ILM intact (as in fig 3D). In such a case, the "ILM" on the ERM detected histologically may simply represent original basement membrane material (fig 3E). In the past, we have shown that both epiretinal and retina cells are capable of synthesising basement membrane components. ${ }^{16}$ Retinal pigment epithelial cells have been shown to produce Bruch membrane laminins. ${ }^{17}$ We speculate therefore that the surgical plane for ERM peel is always internal to the ILM. This would account for the fact that

- there are never any holes or defects visible clinically in the ILM during surgery;

- double peeling seems to be feasible in all cases in this series. However, just because it is feasible does not mean that it is justifiable or sensible. A second peel may cause complications or further damage as a result of the added manipulation.

Our results showed that ILM specimens often contained ERM (six of 16) or cellular remnants (six of 16) on the vitreous surface of the ILM. A possible explanation for the presence of ERM on the ILM could be that the area of ILM excision was larger than the area from which ERM was peeled, in which case the presence of ERM would not have been surprising; ERM would simply be at the edge of the ILM removed. The alternative explanation is of more concern: that the conventional way of peeling ERM leaves fragments or island of cells behind on the ILM and that these residual tissues could form the nidus for reproliferation. Had we found no residue on the vitreous surface side of the ILM, there would be no reason for removing the ILM as a separate peel. Because we found cells adherent to the ILM, the findings support the assertion that removal of ILM in macular pucker might theoretically lead to a more complete removal of epiretinal elements.

Ultimately, the value of routinely ILM peeling after ERM peeling needs to be tested using a randomised trial. Clinically, such a trial is relevant not only with regards to the issue of reproliferation and recurrence of ERM, but also with regards to whether a better quality of vision can be achieved by excising the ILM. At present, there is no easy way of quantifying metamorphopsia. The quality of the vision could be an improvement because there was less distortion, and this improvement might not be reflected by simply measuring the visual acuity. Despite seemingly complete and adequate removal of ERM, some patients with macular pucker continue to complain of slight metamorphopsia or distortion afterwards. It remains uncertain if these symptoms might be due to very fine reproliferated ERM. Richter-Mueksch et al ${ }^{18}$ have recently shown that microperimetry could detect improvements in visual function after macular pucker surgery beyond what can be demonstrated by visual acuity measurements. OCT studies before and after surgery for macular pucker have shown that the macular thickness decreased after ERM surgery, but the macular profile rarely returned to normal. ${ }^{19}$ It would be interesting to know if routine ILM peeling helps reduce the thickness of the macula to achieve a better anatomical outcome and hopefully a better functional result.

The routine peeling of ILM over a wide area at the macula has been demonstrated to be largely harmless in macular hole surgery, and because of macular hole surgery, ILM peel has become a routine skill in the vitreoretinal surgeons repertoire. Our study has shown that surgically, "double peel" for macular pucker is possible in every case using TB. Most likely, this is because the surgical plane of dissection is internal to native ILM.

\section{Competing interests: None.}

Ethics approval: Approval of the hospital ethics committee was obtained for the study including permission for the collection and analysis of histological samples.

Patient consent: Informed consent was obtained from each patient.

\section{REFERENCES}

1. Livingstone $\mathbf{B I}$, Bourke RD. Retrospective study of macular holes treated with pars plana vitrectomy. Aust N Z J Ophthalmol 1999;27:331-41.

2. Schrader WF, Schrenker M. Can the functional outcome in macular hole surgery be improved by internal limiting membrane maculorhexis? Klin Oczna 1999;101:169-73.

3. Uemura A, Kanda S, Sakamoto $Y$, et al. Visual field defects after uneventful vitrectomy for epiretinal membrane with indocyanine green-assisted internal limiting membrane peeling. Am J Ophthalmol 2003;136:252-7.

4. Sivalingam A, Eagle RC Jr, Duker JS, et al. Visual prognosis correlated with the presence of internal-limiting membrane in histopathologic specimens obtained from epiretinal membrane surgery. Ophthalmology 1990;97:1549-52.

5. Kwok AK, Lai TY, Li WW, et al. Indocyanine green-assisted internal limiting membrane removal in epiretinal membrane surgery: a clinical and histologic study. Am J Ophthalmol 2004;138:194-9.

6. Park DW, Dugel PU, Garda J, et al. Macular pucker removal with and without internal limiting membrane peeling: pilot study. Ophthalmology 2003;110:62-4.

7. Smiddy WE, Maguire AM, Green WR, et al. Idiopathic epiretinal membranes: ultrastructural characteristics and clinicopathologic correlation. Retina 2005;25: 811-20.

8. Gibran SK, Prime W, Cazabon J, et al. Successful retrieval of internal limiting membrane specimens. Eye 2007;21:298-9.

9. Hiscott PS, Grierson I, McLeod D. Natural history of fibrocellular epiretinal membranes: a quantitative, autoradiographic, and immunohistochemical study. Br J Ophthalmol 1985;69:810-23.

10. Haritoglou C, Schumann R, Reiniger I, et al. Evaluation of the internal limiting membrane after conventional peeling during macular hole surgery. Retina 2006;26:21-4.

11. Foos RY. Vitreoretinal juncture-simple epiretinal membranes. Albrecht Von Graefes Arch Klin Exp Ophthalmol 1974;189:231-50.

12. Posselt D, Rahman R, Smith M, et al. Visual outcomes following ICG assisted ILM peel for macular hole. Eye 2005;19:279-83.

13. Haritoglou C, Gandorfer A, Gass CA, et al. Histology of the vitreoretinal interface after staining of the internal limiting membrane using glucose $5 \%$ diluted indocyanine and infracyanine green. Am J Ophthalmol 2004;137:345-8.

14. Li K, Wong D, Hiscott P, et al. Trypan Blue staining of internal limiting membrane and epiretinal membrane during vitrectomy: visual results and histopathological findings. Br J Ophthalmol 2003;87:216-9.

15. Wilkins JR, Puliafito CA, Hee MR, et al. Characterization of epiretinal membranes using optical coherence tomography. Ophthalmology 1996;103:2142-51.

16. Hiscott P, Sheridan C, Magee R, et al. Matrix and the retinal pigment epithelium in proliferative retinal disease. Prog Retinal Eye Res 1999:167-90.

17. Aisenbrey S, Zhang M, Bacher D, et al. Retinal pigment epithelial cells synthesize laminins, including laminin 5, and adhere to them through alpha3- and alpha6containing integrins. Invest Ophthalmol Vis Sci 2006;47:5537-44.

18. Richter-Mueksch S, Vecsei-Marlovits PV, Sacu SG, et al. Functional macular mapping in patients with vitreomacular pathologic features before and after surgery. Am J Ophthalmol 2007;144:23-31.

19. Massin P, Allouch C, Haouchine B, et al. Optical coherence tomography of idiopathic macular epiretinal membranes before and after surgery. Am J Ophthalmol 2000;130:732-9. 